Sociologie et sociétés

SOCIOLOGIE

ETSOCIÉTÉS

\title{
Le professionnalisme dans les services : un secours abrutissant
}

\section{John McKnight}

Volume 9, numéro 1, avril 1977

La gestion de la santé

URI : https://id.erudit.org/iderudit/001095ar

DOI : https://doi.org/10.7202/001095ar

Aller au sommaire du numéro

Éditeur(s)

Les Presses de l'Université de Montréal

ISSN

0038-030X (imprimé)

1492-1375 (numérique)

Découvrir la revue

Citer cet article

McKnight, J. (1977). Le professionnalisme dans les services : un secours

abrutissant. Sociologie et sociétés, 9(1), 7-19. https://doi.org/10.7202/001095ar

\section{Résumé de l'article}

Plus les économies des nations modernes sont basées sur la production de services plutôt que de biens, plus la croissance économique exige une augmentation du besoin pour les services. Les idéaux humanistes qui sont associés à la fourniture de services camouflent les implications politiques de ce besoin économique. Les effets cachés de cette production de besoins inhibent toujours davantage la capacité des individus d'agir et la capacité des communautés de résoudre leurs problèmes. On crée ainsi des sociétés qui ont besoin de clients plutôt que l'on crée des communautés qui seraient les expressions publiques des projets collectifs. 


\section{Le professionnalisme dans les services: un secours abrutissant*}

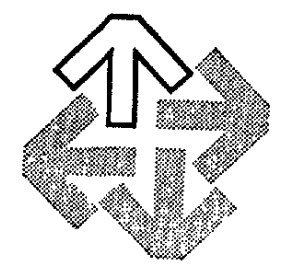

JOHN MCKNIGHT

Ce que les sociétés modernes vendent c'est du service. On s'en aperçoit dans le langage actuel. On parle de la productivité de l'enseignement, des consommateurs de soins et de l'industrie juridique. Ce sont des intrants (inputs) psychologiques et des extrants (outputs) familiaux. L'économiste raffiné parle de marchés et non de clients.

C'est aussi dans la manière de compter que l'on peut voir qu'on est dans un marché de services. Il y a les unités servies et celles qui procurent les services. Et ce qui est encore plus significatif, le Produit National ou Mondial Brut peut se décompter en pourcentage selon les services " fournis".

Les sociétés modernes, socialistes ou capitalistes, se signalent toutes par le fait que dans le calcul de leur Produit National Brut, un pourcentage de plus en plus élevé va aux services. Cette troisième étape du développement économique se distingue des autres par son potentiel illimité. Les limites imposées par les ressources naturelles, le capital et la terre ne peuvent, en principe, absolument pas restreindre une production de services comme elles pouvaient le faire dans le cas d'une production de marchandises. Par conséquent, le marché des services peut se développer à l'infini car, apparemment, il n'y a pas de limites aux besoins pour lesquels on fabrique du service ${ }^{1}$.

\footnotetext{
*Texte présenté au premier symposium du Centre de Bioéthique de l'Institut de Recherches Cliniques de Montréal. Traduction: Madeleine Robert.

1. Gordon, A. et al., "Beyond Need: Toward a Serviced Society ", Crimini Di Pace, Nuova Politecnico, Torino, 1975, disponible de l'auteur, Center for Urban Affairs, Northwestern University, Evanston, Illinois.
} 
Ainsi, la meilleure manière de définir les nations modernes, ce serait par leur économie de services. Socialement parlant, ce sont des sociétés de services. Individuellement parlant, elles sont peuplées de producteurs de services et de consommateurs de services - de professionnels et de clients.

La politique des sociétés de service se décante peu à peu. D'une part, les budgets publics ont de plus en plus de mal à supporter le poids des services. Un grand nombre de gouvernements nationaux et locaux se voient obligés de prendre des décisions politiques tout à fait nouvelles, qui consistent à trancher entre deux services concurrents. Faut-il soutenir davantage l'enseignement et moins la médecine? Et à l'intérieur de chaque secteur des services des dilemmes se posent de façon aussi insoluble. Vaut-il mieux que les taxes défrayent davantage les avortements ou le vaccin contre la grippe?

D'autre part, ces dilemmes sont souvent noyés dans l'idéologie apolitique du service. La vieille politique, fondée sur une économie de marchandises, permettait de débattre civiquement des priorités du pays en terme de blé, d'acier, d'automobiles ou d'habitations.

Ce dont la nouvelle politique doit discuter, c'est de savoir s'il vaut mieux augmenter le nombre des médecins, celui des enseignants, celui des avocats ou celui des travailleurs sociaux. Autrement dit, doit-on échanger de la santé cop̧tre de l'enseignement ou de la justice contre le bien-être de la famille? Il est impossible d'envisager ces choix selon une politique traditionnelle. Alors qu'autrefois, il était possible de choisir entre le blé et l'acier, il est apparemment impossible de prendre une décision politique en faveur de la santé ou de l'enseignement, parce qu'en fait on ne peut pas choisir entre les deux: ce sont des services. En effet, les services sont tellement en dehors de tout débat politique que la plupart des gouvernements finissent par résoudre le dilemme en décidant qu'il y aura moins de blé et davantage d'enseignement, moins d'acier et davantage de médecine.

Ce qui ne veut pas dire que ces choix sont justes ou même que les termes de l'alternative sont pertinents. Cela signifie plutôt que les services semblent par nature tellement apolitiques que le public, et ceux qui font la politique, ont du mal à reconnaitre que les services représentent l'enjeu politique essentiel de nombreuses économies modernes.

Le référent symbolique du service nous fera mieux comprendre l'immunité politique des services.

On paie pour recevoir un service. La "marchandise " que l'on paie c'est la sollicitude. La sollicitude (care) est un acte d'amour. Nous disons « je me soucie d'elle plus que de tout au monde » ou bien « je prends soin de ma mère et de mon père ".

L'amour c'est la sollicitude. La sollicitude payante c'est du service. Inversement, le service c'est la sollicitude, c'est-à-dire l'amour.

L'amour c'est la valeur universelle, apolitique. Donc la nature apolitique du service découle de son assimilation à l'universalité et à l'infinité de l'amour. Demandez à n'importe quel employé des services ce qui en fin de compte lui plaît vraiment dans son travail, la plupart du temps il vous répondra quelque chose comme c'est de me soucier des gens et de les aider. Insistez et la réponse de l'individu sera en général qu'il " aime les gens $»$.

Comme l'amour n'est pas un enjeu politique, la sollicitude n'est pas un problème politique et l'entreprise du service est la seule à produire des « biens " apolitiques, infinis, incontestables. 
Cette analyse pourrait sembler beaucoup trop symbolique, considérons cependant le langage politique utilisé dans ce domaine aux États-Unis. Le premier grand programme d'assurance-maladie offert par l'État ne s'est pas présenté comme un acte politique visant à faciliter l'accès au système médical et à augmenter les profits de ce dernier. On l'a appelé Medicare.

Le président de la American Federation of Teachers a, dans une récente entrevue télévisée ${ }^{2}$, signalé aux membres qu'un nombre sans cesse croissant d'enseignants étaient en chômage et que ceux qui étaient en train d'obtenir leurs diplômes des institutions de préparation à l'enseignement constituaient un nouveau et immense réservoir d'enseignants. Devant ce sérieux problème économique, il mentionna que d'importants secteurs de la société avaient besoin de l'enseignement: la population préscolarisée, les adultes et les personnes âgées. Et pour répondre à ce besoin, il réclamait un nouveau programme gouvernemental qui garantirait à vie à tout Américain le droit à l'instruction. Il appelait ce programme Educare.

Les étudiants en droit représentent, aux États-Unis, $40 \%$ du nombre d'avocats en exercice. Dans une étude récente ${ }^{3}$, on demandait aux membres les plus en vue du barreau américain, quelles mesures ils suggéraient pour que les futurs avocats qui allaient inonder le marché, puissent assurer des services et être payés en conséquence. La réponse la plus souvent obtenue était qu'il fallait provoquer le besoin d'un programme soutenu par l'État, qui assurerait à tous le droit à des services juridiques. Ce projet a reçu unanimement le nom de Judicare.

Il est donc évident que le mot « soin » est un puissant symbole politique. Ce qui l'est moins, c'est qu'il masque les intérêts politiques de ceux qui fournissent les services. Le lien symbolique existant entre soin et amour rend la chose encore moins apparente. En conséquence les enjeux politico-économiques des services se camouflent sous un masque d'amour.

Derrière ce masque on trouve tout simplement ceux qui fournissent les services, leurs systèmes, leurs techniques, et leur technologie. Un monde d'affaires en quête de marchés, une économie à la recherche de nouveaux potentiels pour se développer, des professionnels assoiffés de revenus.

Le masque de l'amour n'est pas artificiel. Il exprime tellement le pouvoir extraordinaire des services, que la plupart de ceux qui les rendent n'arrivent pas à distinguer ce masque de leur propre visage.

L'idéologie du service n'est pas une hypocrisie. Être hypocrite c'est faire semblant de vouloir quelque chose. Celui qui actuellement fournit les services croit, peut-être même davantage que celui qui les reçoit, qu'il s'agit de sollicitude et d'amour.

L'idéologie du service n'est pas un complot. Un complot est manigancé par un groupe qui veut obtenir de fructueux résultats. Les efforts conjugués de celui qui actuellement fournit des services et de ses compagnons visent des résultats soi-disant bénéfiques. Les masques sont de chair.

Pour séparer le masque de la chair, il faut se pencher sur un autre symbole - le besoin. On dit qu'amour est besoin; qu'on a besoin de sollicitude. Les services sont des

2. Shanker, A., Television interview between Albert Shanker, President, American Federation of Teachers and Edwin Newman, p. 19 du manuscrit, sans date.

3. Mincberg, E., "The Legal Profession and the Demand for Legal Services », miméographié, Center for Urban Affairs, Northwestern University, 1973. 
besoins. Ceux qui les rendent comblent ces besoins. Les gens forment des agglomérats de besoins. La société éprouve des besoins. L'économie doit être organisée selon ces besoins.

Si le besoin est un manque de quelque chose, il faut alors pour séparer le masque de la chair se demander: de quoi manque-t-on?

Pour certains, nous manquons de docteurs, d'enseignants, d'avocats, de travailleurs sociaux et de psychiatres.

Pour d'autres, notre santé, notre instruction, notre justice, notre bien-être social et mental sont déficients.

Dans une société «moderne " dont l'entreprise essentielle est le service, la réponse politique réaliste serait que nous manquons surtout de revenus suffisants pour payer adéquatement les professionnels et développer l'économie qu'ils nous laissent pressentir.

Les masques de l'amour et de la sollicitude éclipsent de sérieux enjeux politiques de nos sociétés " modernes » - l'exigence de fabriquer des besoins afin de rationaliser une économie de services. Alors, on peut considérer que Medicare, Educare, Judicare, Socialcare, et Psychocare sont des systèmes destinés à combler les besoins de ceux qui fournissent les services et des économies qu'ils engendrent.

Si on retire le masque de l'amour, on voit le visage de celui qui fournit les services et son besoin de revenus et on découvre un système économique qui a besoin de se développer. À partir de là, le client est moins quelqu'un qui a des besoins que quelqu'un dont on a besoin. En langage d'affaires, le client est moins consommateur que matériau brut du système de services. En langage administratif, le client devient à la fois extrant et intrant. Son rôle essentiel est de combler les besoins de ceux qui fournissent les services, du système de services et de l'économie nationale. L'habilité des professionnels du service à fabriquer des besoins qui permettent à l'économie du système de services de se développer, devient alors le souci politique numéro un.

Si cette analyse est juste, les jugements de valeurs sont hors propos. Après tout, une société de services fournit effectivement une économie, une structure d'organisation sociale, et des travailleurs stimulés par des valeurs éthiques: amour et sollicitude. Si ces dernières correspondent à des besoins légitimes, alors on peut adhérer au marché du développement, de la croissance, de la création, de la recherche et de la fabrication des services, sans être obligé pour autant de mettre les besoins des professionnels sur le dos des citoyens. Leurs besoins peuvent s'envisager en termes politiques et économiques sans les recouvrir du masque apolitique de l'amour.

Cette décision politique poserait cependant un problème dans la réalité politique. Dans toutes les sociétés « modernes ", les citoyens soulèvent d'inquiétantes questions, les voici en langage populaire: pourquoi investissons-nous autant dans la médecine alors que notre santé ne s'améliore pas? Pourquoi investissons-nous autant dans l'enseignement alors que nos enfants semblent être moins instruits? Pourquoi investissons-nous autant dans des systèmes de justice criminelle alors qu'il est de plus en plus dangereux de vivre dans nos sociétés et qu'elles semblent de moins en moins égalitaires? Pourquoi investissons-nous autant dans la santé mentale alors que les maladies mentales sont plus fréquentes? Comme si ces questions populaires n'étaient pas suffisamment inquiétantes, un nouveau groupe de critiques du système des services se demande si nous ne sommes pas en train d'investir toujours davantage pour obtenir, en fait, l'inverse de ce pourquoi le 
système est fait ${ }^{4}$. Le problème serait mieux posé si on parlait de iatrogenèse - de maladies créées par les docteurs. La question que pose cette nouvelle critique n'est pas de savoir si les services diminuent alors que les investissements augmentent, mais plutôt de savoir si nous n'aboutissons pas à l'inverse de ce que le système des services est censé «produire».

Le nouveau problème est de savoir si en améliorant la médecine on n'obtient pas davantage de maladies? Si en augmentant le nombre des avocats et des policiers on n'obtient pas davantage d'injustices et de crimes? Si en augmentant le nombre d'écoles et d'enseignants on n'obtient pas davantage d'ignorants? Si en augmentant le nombre de travailleurs sociaux on n'obtient pas encore plus de foyers brisés?

Ce problème est celui qui remet le plus en question les systèmes des services qui, auparavant, demeuraient apolitiques. En effet lorsque les services incarnent la sollicitude et l'amour, ils servent de tremplin politique; lorsqu'on reconnaît qu'ils sont moins efficaces qu'avant, ils deviennent des atouts politiques; et même s'il est possible politiquement de retirer le masque de l'amour et d'admettre que les services sont des systèmes en quête de ressources pour permettre aux économies de se développer, il est politiquement impossible de conserver une économie de services si les gens s'aperçoivent que le système des services fait plus de mal que de bien et que l'aide fournie par des services professionnels peut être abrutissante.

Depuis quelques années, les meneurs d'avant-garde de l'entreprise de services se sont rendu compte de la menace de iatrogenèse. Ils commencent à mettre en place de nouvelles stratégies pour faire face à la contre-productivité du système des services. Fondamentalement leur réaction a été de faire appel à d'autres professionnels - les administrateurs. Ils supposent que si les services professionnels sont incapables de contrôler leur iatrogenèse, alors l'administrateur professionnel peut s'ériger en réformateur moderne, contrôler et diriger les systèmes de façon à neutraliser cette contre-productivité et à préserver le soutien politique nécessaire au développement du système des services.

La nouvelle administration des services, appliquant l'art d'administrer du secteur de production des marchandises à la rationalisation du système des services, découvre quatre éléments susceptibles d'être manipulés: les budgets, le personnel, la structure de l'organisation et la technologie. Ainsi donc, l'administrateur du service s'occupe activement à institutionnaliser des systèmes de contrôle comptable, à développer des systèmes de formation du personnel, à restructurer les systèmes de distribution et à implanter de nouvelles technologies. Les administrateurs les plus avant-gardistes ont employé les techniques de mise en marché les plus raffinées pour arriver à une cinquième manipulation - préparer le client. Ils se rendent compte que si le besoin d'un service n'existe pas, il est possible de le fabriquer. Si la conception populaire d'un besoin ne correspond pas au service, les administrateurs modernes ont à leur disposition des techniques de mise en marché capables de persuader les gens de s'adapter au service.

Les techniques d'une administration professionnelle vont-elles stabiliser le marché des services en limitant les effets de iatrogenèse?

Le potentiel des systèmes d'administration moderne est impressionnant. On pourrait très bien imaginer que l'action combinée de ce potentiel et de l'idéologie apolitique du service constitue une force irrésistible qui tracera la voie des économies modernes.

4. Illich, I., Némésis médicale: l'Expropriation de la santé, Paris, Seuil, 1975. 
Par ailleurs, on peut aussi penser qu'il y aura un contre-poids - en fait une chose immuable - qui interviendra contre cette force irrésistible. Si une telle chose existe, c'est qu'elle est "produite " par les limites inhérentes à des services bien administrés.

Dans les pages qui suivent, nous voudrions cerner les effets intrinsèquement abrutissants des rapports engendrés par les services modernes, et évaluer les conséquences politiques de leur développement.

L'abrutissement associé à la professionnalisation du service naît d'un ensemble de conceptions sur la nature du besoin. De ces conceptions, dérive un ensemble de postulats sur les remèdes adéquats.

\section{EFFET DU PROFESSIONNALISME SUR LA CONCEPTION DU BESOIN}

Si l'on considère tout d'abord la conception «professionnaliste " du besoin, on constate que ses effets abrutissants proviennent de trois éléments principaux.

Premièrement, le besoin se définit comme manque. Un besoin pourrait s'entendre comme un état, un désir, un droit, un dû, un fantasme ou comme un problème insoluble.

La pratique professionnelle définit systématiquement le besoin comme un manque fâcheux pour soi ou comme un vide chez autrui. Ce qui rappelle la devinette pour enfant, qui consiste à faire décrire un verre rempli à moitié d'eau: est-il à moitié plein ou à moitié vide?

Le rôle essentiel du professionnalisme moderne consiste à justifier la vision de ceux qui considèrent leur voisin comme à moitié vide. Par apprentissage, les professionnels sont arrivés à développer un système ultra-complexe, d'un superbe niveau de raffinement, pour étiquetter les différentes manières de percevoir ce vide. La recherche professionnelle se consacre de plus en plus à élever le rebord du verre, afin d'être sûr qu'il ne sera jamais rempli - même par des "services compétents".

Dans une économie de services où la plupart des revenus proviennent de "l'aide * professionnelle qui est fournie, les nations ont un besoin toujours grandissant d'individus déficients. Ainsi une société qui prétend combler des besoins définis comme des déficiences personnalisées se comprend mieux comme une économie en manque de manques. Par un gauchissement comique, on pourrait voir des sociétés de voisinage où pour avoir des revenus il faudrait que chacun dépiste les déficiences des autres. Le résultat serait que le voisinage ainsi conçu ne pourrait plus être une communauté agissante, capable de comprendre un problème et de le résoudre.

Le deuxième facteur d'abrutissement lié à la définition «professionnaliste » du besoin consiste à localiser la déficience dans le client. Alors que la plupart des professionnels «modernes " admettent que tout problème individuel prend racine dans le contexte socio-politique, les solutions offertes en général par les services, elles, isolent les individus du contexte. Cette séparation déforme la vision que les professionnels ont de leur propre contexte: Comme leurs outils et leurs techniques-remèdes sont en général limités à des interactions individuelles, bien entendu le besoin s'interprète lui aussi en fonction de l'individu. Ce sont les outils qui définissent le problème et non l'inverse.

Une étude récente sur les enfants en institution en fournit un exemple ${ }^{5}$. Ces enfants ont été enlevés à leurs parents parce que ceux-ci avaient été jugés incapables de s'en

5. Gordon, A. et al., "Experiences of Wardship: Interim Report II ", Center for Urban Affairs, Northwestern University, mars 1976. 
charger comme il faut. Ces enfants ont par conséquent été placés dans des institutions spécialisées. Néanmoins, dans la majorité de leurs dossiers, on parle toujours de l'enfant comme le problème.

Par ailleurs, les autorités qui ont permis que l'enfant soit retiré de sa famille, reconnaissent qu'en général c'est parce que la famille était pauvre. Toutefois, ils ne pouvaient leur fournir aucune aide économique. Les institutions spécialisées, par contre, avaient beaucoup de ressources. Le système des services comble ses besoins économiques en institutionnalisant un problème conçu comme étant individuel. On pourrait dire que la conséquence négative en est que les familles se sont appauvries encore davantage, à cause des dépenses occasionnées par les "soins» des services professionnels. En terme de iatrogenèse, le système des services " produit » des familles brisées.

La définition du besoin, thérapeutique et individualisante, se voit contrée par des mouvements de "libération ». C'est ce que font les mouvements pour les droits civiques et ceux de libération de la femme. L'essentiel de leur rôle idéologique consiste à convaincre les minorités et les femmes de leur finalité d'être humain à qui il ne manque rien et qui ne dépend pas de systèmes qui soi-disant satisferaient leurs besoins par une aide professionnelle individualisée. Au contraire, ces mouvements, en poussant à l'action collective, se battent pour que soit dépassée cette « psychologie » centrée sur l'individu et la déficience.

Le troisième facteur essentiel d'abrutissement, dû à la définition «professionnaliste » du besoin, provient de la spécialisation — «produit» le plus fondamental des systèmes de techniques et de technologie avancées. Tout le monde sait que la spécialisation engendre des systèmes de services extrêmement compartimentés, à l'organisation très complexe, ce qui permet aux administrateurs de se pencher sur de superbes problèmes d'organisation. On consacre d'incroyables ressources humaines et financières à la rationalisation de ces systèmes, soulevant ainsi des problèmes politiquement acceptables et justifiant la croissance de l'économie par les services.

Ce que l'on saisit beaucoup moins, c'est qu'alors les citoyens reflètent ces systèmes. Puisque ces systèmes sont divisés en sections administratives, obligatoirement le client est lui aussi considéré et traité comme un ensemble de parties à administrer, chacune d'entre elles correspondant à une mécanique particulière de services.

La complexité des systèmes de services nous fait penser aux dessous de plats de certains restaurants, où l'on voit le dessin d'une vache découpée en steak, rôti, côtes et langue.

De la même manière, les services professionnels parlent de l'homme comme une série de morceaux. Nous avons besoin non seulement d'un pôdiatre pour nos sabots, et d'un oto-rhino-laryngologiste pour notre museau. Mais notre temps, lui aussi, est découpé en morceaux par le professionnalisme « moderne ». À présent, les services professionnels nous persuadent que, pour chaque âge, il existe un ensemble de besoins correspondants. Les professionnels ont " découvert » que de l'enfance à la vieillesse nous passons par sept moments de crise et que pour chacune d'entre elles, il faut une aide professionnelle. Elizabeth Kubler-Ross va encore plus loin en découvrant cinq étapes dans la mort. À cause de cette révélation, on va avoir une nouvelle série de professionnels, et de l'aide pour l'étape numéro un de la mort, numéro deux, etc. À ces thérapeutes de la mort, s'ajouteront les chercheurs professionnels chargés de découvrir pourquoi certaines personnes sautent des étapes. 
La définition du besoin donnée par les services professionnels rend la notion de personne de plus en plus rudimentaire. L'individualisation du besoin amputait déjà les gens de leur contexte social et de la possibilité d'une prise en charge collective des problèmes, la simplification de la personne élimine jusqu'à la probabilité d'une action individuelle. Ce qui reste ce sont des gens découpés en besoins, dans le temps et dans l'espace. Heureusement qu'ils peuvent quand même être rassemblés pour former une unité humaine encore capable de payer les services reçus.

En bref, les services professionnels définissent le besoin comme une déficience et l'individualisation et la simplification de la personne s'en suivent. Dit en termes personnels, le système des services nous affirme trois choses:

Vous qui êtes déficient

C'est vous le problème

Vous avez une série de problèmes.

Replacés dans la perspective des intérêts du système des services, ces postulats deviennent:

Nous avons besoin de déficiences

L'unité économique dont nous avons besoin, c'est l'individu

L'unité économique optimale dont nous avons besoin c'est un individu à déficiences multiples.

\section{EFFET DU PROFESSIONNALISME SUR LA CONCEPTION DU REMEDDE AU BESOIN}

Ces conceptions de la nature du besoin entraînent automatiquement et logiquement une série de postulats quant aux remèdes appropriés, chacun provoquant des effets intrinsèquement abrutissants.

Le premier de ces postulats renvoie à la définition individualisante du besoin. Si c'est vous le problème, alors c'est moi, le professionnel de l'aide, qui suis la solution.

Ce n'est pas vous la solution, ni vos semblables. Ce n'est pas non plus dans le contexte social et économique qu'elle se trouve. Mais cette solution existe.

Je suis la solution

Le postulat essentiel est que le service est un échange unilatéral.

Bien entendu, grâce à la sensibilité de certains professionnels, tout un ensemble spectaculaire de mécanismes spécialisés ont été mis en place afin de réfuter la nature unilatérale des services. On les nomme services aux groupes, services aux groupes de pairs, services au client, services aux communautés. Chacune de ces dénominations tente de satisfaire symboliquement les besoins de la gent du service qui elle a besoin de nier la nature unilatérale de ses échanges.

Bien sûr certains professionnels cherchent, par humanisme, à définir leur rôle de façon démocratique, mais à part l'argent déboursé par le client et le sentiment qu'a le professionnel d'avoir aidé ce dernier, il est difficile de trouver autre chose de bilatéral. De fait, la "faute professionnelle " la plus élémentaire consiste à permettre « l'échange réciproque " avec un client. Être professionnel, c'est tenir ses distances de façon à ce que le client comprenne bien qui, dans la relation, reçoit un service. 
Malgré cette façade démocratique, il est évident que l'effet d'abrutissement dû à l'aide unilatérale des professionnels vient du postulat implicite: "vous irez mieux parce que moi j'en sais davantage ».

Ce sont de telles présuppositions qui politiquement deviennent la base des systèmes anti-démocratiques. Il est possible qu'en effet ces sociétés, fondées sur des économies de services unilatéraux, soient en train de conditionner systématiquement les gens à avoir des dirigeants anti-démocratiques qui pourront miser sur l'esprit de dépendance engendré par des rapports unilatéraux avec les experts de l'aide professionnelle, qui apprennent aux gens que: "vous irez mieux, parce que je m'y connais ».

Le deuxième postulat abrutissant de la conception professionnelle du remède, c'est que le remède définit le besoin. Les systèmes de services exigent que soient utilisés de toute urgence leurs techniques toujours plus attrayantes, ainsi que leurs outils toujours plus merveilleux.

Le problème c'est qu'il n'est pas facile de faire comprendre au public " l'intérêt » de ces beaux outils, brillants et compliqués. C'est ainsi qu' on peut voir les professions suivre leur propre logique et créer des systèmes de mise en marché pour justifier l'usage de ces outils, sous-entendu que le client 'ne peut pas comprendre ce dont il a besoin. S'il veut profiter des avantages des soins professionnels, alors il doit aussi admettre que c'est le professionnel qui sait ce dont il a besoin.

Ainsi les outils professionnels deviennent la justification du pouvoir des professionnels sur la définition du besoin, décidant non seulement du remède adéquat mais des termes mêmes du problème. De plus en plus les professionnels estiment que pour combler les déficiences, ils doivent aussi avoir le privilège de les déterminer.

Bien entendu, il n'y a pas de plus grand pouvoir que celui de définir le problème. Ce pouvoir permet de limiter la liste des solutions appropriées. Si celui qui fournit le service peut effectivement imposer sa propre vision du problème, alors il a le pouvoir de décider des besoins du voisin plutôt que de lui en laisser l'initiative.

Non seulement ce pouvoir permet aux professionnels d'utiliser leurs beaux remèdes tout neufs mais il implique également que les citoyens sont des gens incapables de savoir s'ils ont un problème et encore moins comment le résoudre.

Les sociétés « civilisées » sont saturées de besoins qui orientent les recherches et les mises en marché. Les professionnels ont « découvert » depuis peu de temps de nouvelles sphères de besoins pour leurs outils: enfants maltraités, difficultés d'apprentissage et traumatismes de sevrage familial (les enfants sont traumatisés parce qu'on les a enlevés à leurs familles qui elles-mêmes étaient soi-disant traumatisantes). Ils ont alors besoin de thérapie. Dans un article récent ${ }^{6}$, Brigitte Berger imagine que, vu le surplus de dermatologues, la calvitie sera très bientôt considérée comme une maladie. Le comble de ce processus d'institutionnalisation est un nouveau programme mis en route par une clinique célèbre des États-Unis ${ }^{7}$. Ce programme offre une chance aux gens qui se sentent " parfaitement bien » de découvrir de façon onéreuse de quoi ils pourraient bien souffrir qui répondrait aux besoins des nouveaux outils.

Lorsque ce sont des professionnels qui ont le privilège de définir un problème alors les citoyens n'existent plus. Ce privilège enlève à ces derniers non seulement la possibilité

6. Berger, B., "Family, Bureaucracy and the "Special Child ", " The Public Interest, Eté 1975, p. 96.

7. Bollinger, R., « Help for the Everyday Problems of Living », Menninger Perspective, automne hiver 1975 , p. $21-23$. 
de décider du problème mais aussi celle de le résoudre. Les affaires politiques sont alors traduites en termes de problèmes techniques et technologiques.

Lorsque la gent professionnelle du service peut définir et le remède $e t$ le besoin, alors la pratique des soins professionnels crée un troisième facteur d'abrutissement. À savoir, le codage des problèmes et de leurs solutions, dans un langage incompréhensible pour les citoyens.

Comme c'est visiblement abrutissant de se faire dire qu'on ne peut pas savoir si on a un problème ou non et lequel, le professionnalisme renforçit le dilemme, en vous démontrant que de toutes façons vous seriez incapable de comprendre ou le problème ou sa solution. Le langage utilisé par les services professionnels nous mystifie sur le problème et sa solution, de telle manière qu'il devient impossible pour le citoyen d'en juger. Les seules personnes " compétentes » qui peuvent décider du mérite de l'opération des services sont les pairs des professionnels, car ils partent tous des mêmes postulats.

Bien que l'on puisse assister à de fascinants conflits de juridiction interne, ceux-ci ne vont rarement jusqu'à briser la règle qui veut que seuls les professionnels comprennent problèmes et solutions. Les conflits internes sont des luttes de pouvoir. Un professionnel qui enfreindrait la règle de dominance professionnelle verrait tous les autres se mettre contre lui.

Le pouvoir du professionnel «moderne" est étroitement contrôlé par ses pairs. L'hérétique est celui qui encourage la prise de conscience du citoyen et transforme sa profession en un échange clair à partir de la volonté du citoyen formulée de façon intelligible.

Le langage codé du professionnel a pour effet d'amoindrir la faculté du citoyen de faire face à la causalité et à ses conséquences. Si je n'arrive à comprendre ni la question ni la réponse, ni le besoin ni son remède, je n'existe que par le bon vouloir des experts $\mathrm{du}$ système. Je ne suis pas dans un monde où je fais et j'agis avec d'autres. Je me trouve plutôt dans un lieu mystérieux, un endroit étrange qui échappe à mon intelligence et à mon contrôle. Seuls les professionnels sont capables de savoir comment tout fonctionne, ce dont $\mathrm{j}$ 'ai besoin et comment me satisfaire. Je suis passif et non actif. J'existe en tant que client et non en tant que citoyen. Ce sont des problèmes techniques et non des systèmes politiques qùi fondent ma vie et ma société.

Une quatrième forme d'abrutissement se développe au fur et à mesure que les professions de service acquièrent le pouvoir de définir unilatéralement le besoin et le code des opérations de service. C'est-à-dire que c'est la gent du service qui apprécie les résultats de leurs propres services.

Sur le plan personnel, cela veut dire que peu à peu le professionnalisme suppose que les citoyens ont besoin de cette unilatéralité parce qu'ils ne savent pas ce dont ils ont besoin et comment se satisfaire. On pourrait au moins penser que les citoyens sauraient apprécier les services qu'ils reçoivent. Ils pourraient être incapables de comprendre leurs problèmes et leurs solutions. Ils pourraient ne jouer aucun rôle dans la définition et dans la résolution des problèmes mais ils pourraient effectivement apprécier les résultats.

C'est cette façon de voir les choses qui anime les mouvements de consommateurs. C'est le dernier bastion du citoyen abruti qui réclame d'avoir au moins le droit de juger des effets ou extrants des services professionnels.

Ce mouvement prétend implicitement que le citoyen se réhabilite en prenant le pouvoir comme consommateur. Cette prétention du mouvement le transforme en fait en 
domestique de la société de service. Il accepte implicitement l'idéologie du service. Les citoyens existent en consommant. Le bien-être du citoyen est défini par une consommation équitable et efficace. Le système de service représente une certaine marchandise. Le rôle du citoyen est d'en évaluer les résultats. Même si les citoyens ne peuvent pas comprendre le système des services, les mouvements de consommateurs supposent qu'ils peuvent au moins savoir si ses effets sont bénéfiques ou non.

Les administrateurs professionnels des systèmes de services ont maintenant affaire avec ce qui reste du citoyen: un consommateur. Alors les professionnels s'occupent de plus en plus à manipuler la façon dont le consommateur va percevoir les résultats des services. Dans un brillant article ayant pour titre "la professionnalisation du client ${ }^{8}$ ", Thomas Dewar montre comment le système médical conditionne les clients à comprendre que s'ils veulent être satisfaits, il faut qu'ils se comportent en clientèle efficace et non comme des gens qui auraient trouvé une solution à leurs problèmes.

L'école est un exemple typique de ce processus. Contrairement aux autres systèmes de services, elle ne cache pas l'institutionnalisation du rôle de client. Le client de l'école est jugé sur la façon dont il satisfait le professionnel. Le fait que c'est le professionnel qui juge son comportement et son rendement est le résultat explicite du système.

Les exigences du professionnalisme généralise l'idéologie de l'école, en répandant l'idée que la réussite vient du comportement comme parfait client. En déniant au client jusqu'à sa capacité de juger d'un résultat, les services professionnels « civilisés » propagent de plus en plus la valeur selon laquelle une clientèle réussie démontre l'efficacité du système. Une fois que la mentalité-client deviendra une valeur essentielle de la société, alors la mentalité-consommateur s'évanouira.

L'idéologie de service atteindra son apogée quand les citoyens vivront dans une économie politique en croyant qu'ils ne peuvent pas savoir s'ils ont des besoins et lesquels, qu'ils ne sont pas capables de comprendre les mécanismes censés satisfaire leurs besoins et qu'ils ne pourront même pas dire s'ils sont satisfaits ou non sauf si les professionnels le décident. Le point final d'une société de services sera mis lorsque le professionnel dira " je suis vraiment satisfait de votre conduite ». L'être civique s'éteindra avec sa réponse: "je vous remercie ».

C'est en ces termes que les conceptions abrutissantes des professionnels se traduisent pour les individus:

Nous sommes la solution à votre problème

Vous ne connaissez pas votre problème

Vous ne pouvez comprendre ni votre problème ni sa solution

Vous n'êtes pas apte à savoir quelle est la bonne solution.

Transposés en termes d'intérêts du système des services et de leurs besoins, ces postulats se transforment ainsi:

Nous avons besoin de problèmes

Nous avons besoin de vous dire lesquels

Nous avons besoin de les résoudre à notre façon

Nous avons besoin que vous soyez satisfait par nos prévenances.

8. Dewar, T., "The Professionalization of the Client », CIDOC, 1974. Disponible de l'auteur, School of Urban Sciences, University of Minnesota, Minneapolis, Minnesota. 
À partir de là, la recherche la plus intéressante et la plus importante, dans nos sociétés « modernes ", est de comprendre les besoins de la gent du service et de ses systèmes. De toute évidence ceux-ci sont importants. Ils fournissent des revenus à la plupart des gens. Ils alimentent les économies nationales. Néanmoins ils n'arrivent jamais à atteindre leurs propres buts, malgré une débauche d'argent et de personnel. Au lieu de produire des " services », ils produisent des professionnels de plus en plus réceptifs mais frustrés, perplexes devant l'inefficacité de l'amour et de la sollicitude à transformer la société et surtout à aider les individus à vivre.

Nos efforts de recherche devraient s'orienter sur les besoins de la gent du service. Après tout, celle-ci constitue la partie la plus importante des populations des sociétés « civilisées »; or elle est de plus en plus triste. C'est une classe de la population qui est aliénée et qui a besoin d'aide, d'attention, de sollicitude et d'amour. Nos sociétés « modernes $*$ ont besoin de trouver comment les aider, tout en réduisant le pouvoir qu'ils ont de dépolitiser la perception des citoyens.

Pour ce faire, nous devrions comprendre les effets politiques de la nature débilitante des définitions du besoin et du remède donnés par les professionnels.

Les services professionnels font partager une vision du monde où nos vies et nos sociétés sont traitées comme une série de problèmes techniques. Cette approche de type technique se dissimule sous le masque de la sollicitude et de l'amour qui occulte les intérêts économiques de la gent du service et l'aspect débilitant de ses pratiques.

Cet abrutissement généralisé, c'est une idéologie qui métamorphose le citoyen en client, les communautés en une juxtaposition d'individus déficients, la politique en un débat pour savoir quel système de services aura la part la plus importante du produit national brut.

Cette analyse ne veut pas prouver la nécessité de transformer le service professionnel afin d'en éliminer les effets abrutissants. Elle veut plutôt démontrer que ces effets sont intrinsèquement liés à la "professionnalisation " «moderne »du service. Quels qu'en soient les résultats bénéfiques, on ne peut rien en dire tant que l'on n'aura pas admis d'abord que ces systèmes poursuivent avant tout leurs propres intérêts, avec tout ce que cela implique comme effets abrutissants. C'est dans cette optique que peut se faire la réhabilitation du citoyen. On peut mettre en balance le taux d'abrutissement et le taux d'éventuels bienfaits dus aux services professionnels. On peut choisir de sélectionner les services dont le taux de bienfaits s'avère supérieur au taux des effets débilitants.

En attendant, la politique des sociétés « modernes " pourrait bien être l'affrontement entre la force irrésistible des entreprises de services et cette force immuable que constitueront les citoyens ayant subi l'abrutissement engendré par l'assistance professionnelle de cette gent privilégiée du service, qui porte un masque d'amour.

\section{RÉSUMÉ}

Plus les économies des nations modernes sont basées sur la production de services plutôt que de biens, plus la croissance économique exige une augmentation du besoin pour les services. Les idéaux humanistes qui sont associés à la fourniture de services camouflent les implications politiques de ce besoin économique. Les effets cachés de cette production de besoins inhibent toujours davantage la capacité des individus d'agir et la capacité des communautés de résoudre leurs problèmes. On crée ainsi des sociétés qui ont besoin de clients plutôt que l'on crée des communautés qui seraient les expressions publiques des projets collectifs. 


\section{SUMMARY}

As modernized nations increasingly base their economies upon the production of services rather than goods, their growth requires an increase in the need for services. The political implications of this economic need are obscured by the humanistic ideals associated with the provision of services. The hidden impacts of need production increasingly disable capacities for individual action and community problem-solving, creating societies in need of clients rather than communities that are civic expressions of citizen purpose.

\section{RESUMEN}

Las economías de las naciones modernas cuanto más están basadas en la producción de servicios que de bienes, el crecimiento económico exige una aumientación mayor de la necesidad para los servicios. Los ideales humanistas que están asociados con el suministro de los servicios, esconden las implicaciones políticas de esa necesidad económica. Los efectos ocultos de esta producción de necesidades inhiben cada vez más la capacidad de los individuos a reaccionar y la capacidad de las comunidades a resolver sus problemas. Se crean así sociedades que tienen necesidad de clientes más bien que de crear comunidades que sean las expreciones públicas de los proyectos colectivos. 